\title{
Borrelia burgdorferi and Treponema pallidum: a comparison of functional genomics, environmental adaptations, and pathogenic mechanisms
}

\author{
Stephen F. Porcella and Tom G. Schwan \\ Laboratory of Human Bacterial Pathogenesis, Rocky Mountain Laboratories, National Institute of Allergy and Infectious Diseases, \\ NIH, Hamilton, Montana, USA \\ Address correspondence to: Tom G. Schwan, Rocky Mountain Laboratories, 903 South 4th Street, Hamilton, Montana 59840, USA. \\ Phone: (406) 363-9250; Fax: (406) 363-9445; E-mail: tom_schwan@nih.gov.
}

Spirochetes are a diverse group of bacteria found in soil, deep in marine sediments, commensal in the gut of termites and other arthropods, or obligate parasites of vertebrates. Two pathogenic spirochetes that are the focus of this perspective are Borrelia burgdorferi sensu lato, a causative agent of Lyme disease, and Treponema pallidum subspecies pallidum, the agent of venereal syphilis. Although these organisms are bound together by ancient ancestry and similar morphology (Figure 1 ), as well as by the protean nature of the infections they cause, many differences exist in their life cycles, environmental adaptations, and impact on human health and behavior. The specific mechanisms contributing to multisystem disease and persistent, longterm infections caused by both organisms in spite of significant immune responses are not yet understood.

The Centers for Disease Control and Prevention reported 16,801 cases of Lyme disease and 37,977 cases of syphilis in the United States in 1998. Syphilis was first observed just over 500 years ago although the bacterial cause was not determined until 1905 (1). Lyme disease was described 25 years ago with its causative agent discovered in late 1981 (2), but progress toward managing Lyme disease has occurred rapidly, and a vaccine for this disease is now approved for human use and is available in the United States (3). This critical advance was made possible in part by the ability to cultivate Lyme disease spirochetes in vitro, due to the prior development of Kelly's medium (4) for cultivating relapsing fever borreliae. In contrast, $T$. pallidum must be grown in the testicles of live rabbits to obtain bacteria for experimental use (5). While no vaccine is yet available for syphilis, the spirochete's obligate association with humans and transmission by sexual contact makes the eradication of this disease at least conceivable. Eradication is not an obtainable goal for Lyme disease, because $B$. burgdorferi is widespread in nature and is maintained in zoonotic cycles involving many species of mammals, some birds, and transmission by small, blood-feeding ticks. Recently, the genome sequences for both of these bacteria were published
(6-8). Here, we compare the biology and genomes of these two spirochetal pathogens with reference to their different host associations and modes of transmission.

Genomic structure

A striking difference between B. burgdorferi and T. pallidum is their total genomic structure. Although both pathogens have small genomes, compared with many well known bacteria such as Escherichia coli and Mycobacterium tuberculosis, the genomic structure of B. burgdorferi

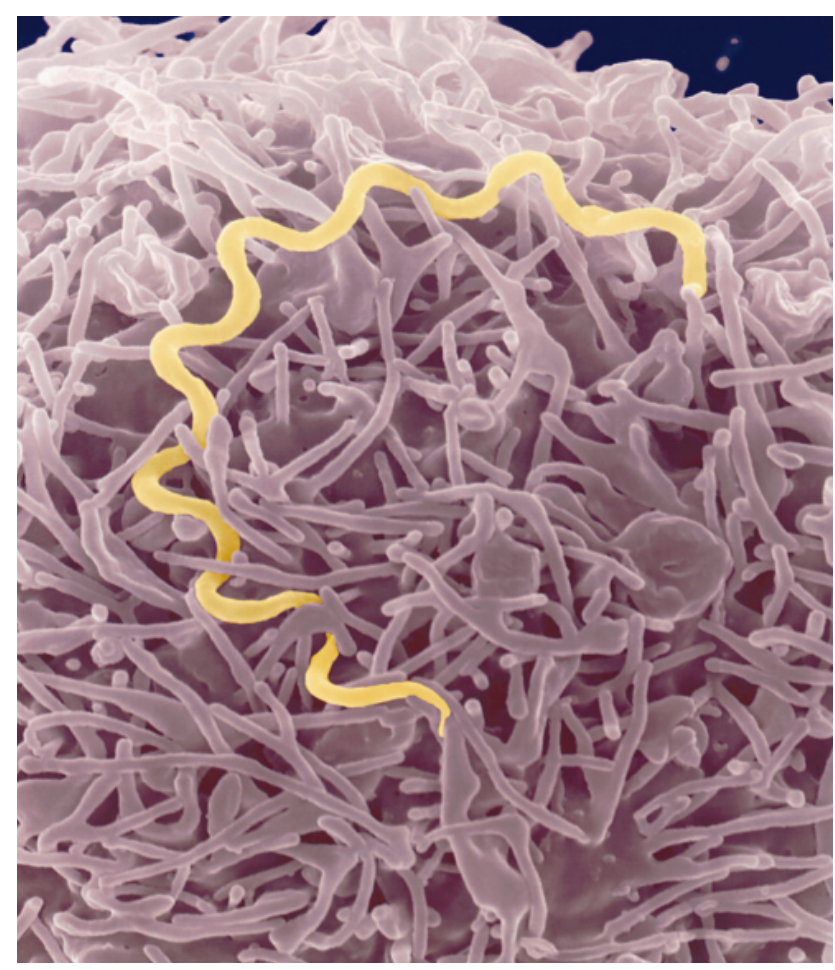

Figure 1

T. pallidum associated with the surface of a human lymphocyte. The scanning electron microscopic image demonstrates the wave-form structure characteristic of spirochetes. 
is one of the most complex known in prokaryotes. This spirochete contains a linear chromosome $910,725 \mathrm{bp}$ in length with an average guanine + cytosine $(G+C)$ content of $28.6 \%$ (6). Twenty-one extrachromosomal elements, including 12 linear and 9 circular plasmids, totaling another 610,694 bp have also been identified (8). There is considerable variation in plasmid content among isolates. Some of these extrachromosomal elements are lost quickly upon serial propagation.

In contrast, T. pallidum contains a single circular chromosome with $1,138,006 \mathrm{bp}$, an average $\mathrm{G}+\mathrm{C}$ content of $52.8 \%$, and no extrachromosomal elements (7), making its total genome approximately $25 \%$ smaller than that of B. burgdorferi. Importantly, none of the 1041 predicted open reading frames (ORFs) of T. pallidum are homologous to any of the 535 intact ORFs or 167 pseudogenes encoded by the $B$. burgdorferi plasmids $(7,8)$. Indeed, over $90 \%$ of the B. burgdorferi plasmid ORFs are unrelated to any known bacterial sequences (8). The novel genes found on the B. burgdorferi plasmids may therefore contribute to the ability of this pathogen to survive and maintain its complex life cycle, alternating between warm-blooded animals and cold-blooded ticks as hosts.

\section{Lipoprotein and pathogen-host interactions}

The $B$. burgdorferi genome contains at least 132 genes encoding putative lipoproteins, but only 22 such genes are present in T. pallidum $(7,8)$. Lipoprotein genes comprise $5 \%$ of the chromosomal ORFs in B. burgdorferi and $2.1 \%$ of those in T. pallidum. The T. pallidum value is closer to that of other types of bacteria such as Helicobacter pylori, in which $1.3 \%$ of its genome encodes lipoproteins $(7,8)$. Interestingly, 14.5 to $17 \%$ of the functionally complete ORFs on the $B$. burgdorferi plasmids encode lipoproteins $(7,8)$. The reasons for this proportionately greater coding capacity for lipoproteins in B. burgdorferi compared with many other bacteria are unknown.

Lipoproteins are the predominant membrane protein immunogens for both T. pallidum and B. burgdorferi (9, 10). No lipoproteins have been definitively localized to the outer surface of T. pallidum. In contrast, the majori- ty of surface-exposed proteins made by $B$. burgdorferi are lipoproteins. Two well-characterized lipoproteins are outer surface protein A (OspA) and OspC. The OspA gene is phylogenetically conserved and its protein product is the immunogen in the recently approved human vaccine for Lyme disease (3). Conversely, the OspC gene has considerable sequence heterogeneity among natural isolates at both the DNA and amino acid level. This gene is also differentially expressed at higher temperatures and its protein product has been hypothesized to be involved with transmission of spirochetes during tick bite and the early colonization in vertebrates (11).

The difference in the number and cellular location of lipoproteins made by the two spirochetes suggests a functional role for the borrelial lipoproteins for survival in diverse hosts and/or transmission by ticks. The synthesis of many borrelial lipoproteins increases when the growth temperature is shifted from $23^{\circ} \mathrm{C}$ to $35^{\circ} \mathrm{C}(11$, 12 ), a change presumed to mimic the warming that occurs when B. burgdorferi passes from ticks to mammals. Consistent with this idea, many B. burgdorferi lipoproteins are recognized by sera from infected animals $(9,12)$, indicating that the mammal is exposed to these proteins during infection. These observations suggest that many of the lipoproteins of B. burgdorferi are involved in host-pathogen interactions, and we assume this to be true as well for T. pallidum.

Given the evidence that borrelial and treponemal lipoproteins interact with the mammalian immune system early in infection, numerous studies have explored their potential role in cytokine production and immune-cell activation. B. burgdorferi outer membrane lipoproteins activate macrophages, endothelial cells, neutrophils, and B cells (13). Dermal inflammation characterized by infiltration of neutrophils, heterophils, and mononuclear cells has been observed in mice and rabbits following injections of lipopeptides of B. burgdorferi and T. pallidum (14). Hence, lipoproteins may be involved in the initial inflammatory processes that occur in erythema migrans and the primary lesion in syphilis.

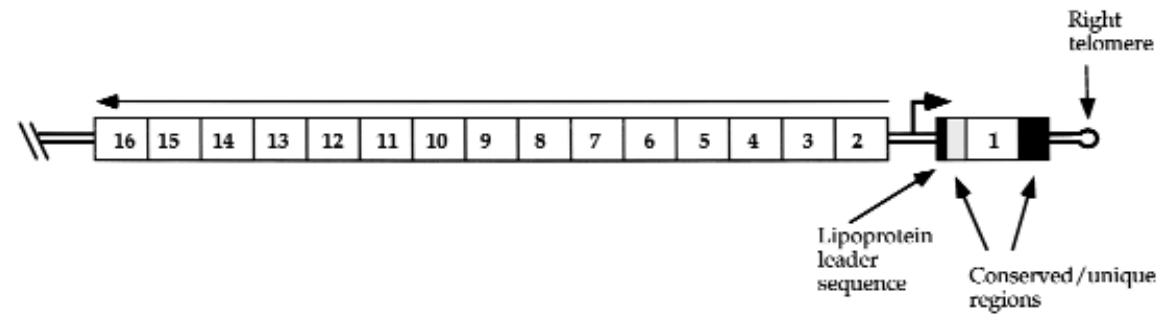

Figure 2

Arrangement of the approximately $10 \mathrm{~kb} v / \mathrm{s}$ locus in B. burgdorferi located near the right telomere of linear plasmid 28-1. The directions of coding or expression are shown with horizontal arrows. Box 1 represents the expressed gene while boxes 2 through 16 represent silent gene cassettes. Figure modified from Zhang et al. (21). 
a
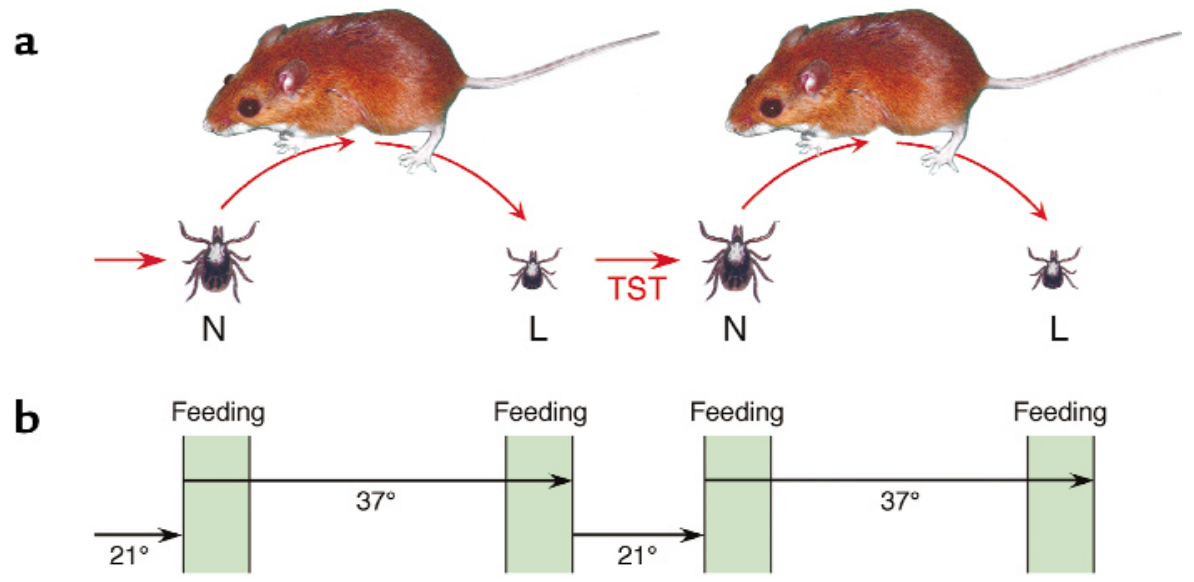

C

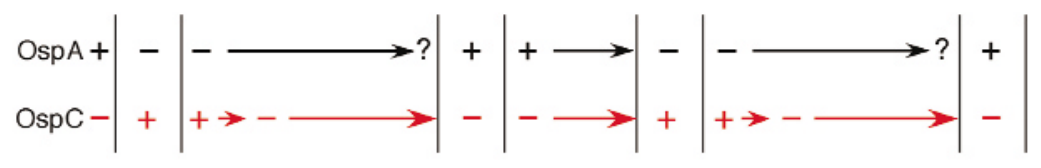

d
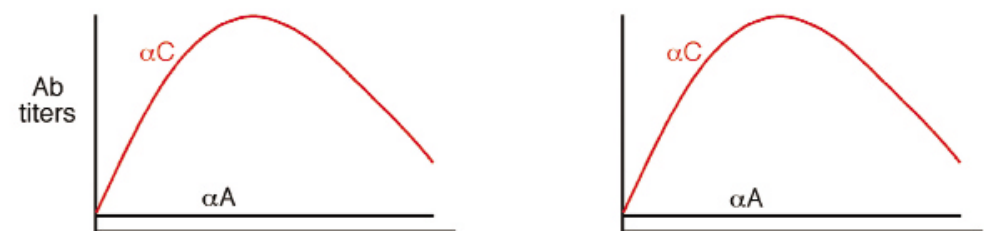

Figure 3

Natural maintenance cycle and associated biological parameters for B. burgdorferi. (a) The spirochete is maintained in nature by alternating infections in white-footed mice and immature lxodes ticks. Larvae ( $\mathrm{L}$ ) acquire spirochetes by feeding on infected mice and maintain the infection through the transstadial (TST) molt. Infected nymphs (N) transmit spirochetes during the next bloodmeal. (b) Spirochetes are exposed to alternating temperatures during tick and mammalian infections, and other parameters associated with tick attachment and feeding. (c) OspA and OspC are differentially expressed by spirochetes during the transmission cycle with alternate tick and mammalian hosts. (d) General trend in antibody titer to OspA and OspC in mice infected by tick bite, supporting the evidence for the lack of OspA expression during infection and the early presence of OspC following the tick bite.

The pathogenesis associated with Lyme disease has stimulated research into the role of spirochete lipoproteins in the disease pathway (15-17). Picomolar amounts of OspA increase CD10 and CD11b, decrease CD62-L, and activate neutrophils (15), suggesting the involvement of lipoproteins in tissue-specific arthritic inflammation, where neutrophils are known to be the predominant infiltrate. The toll-like receptor 2 has been implicated also in signal transduction for borrelial and treponemal lipopeptides and lipoproteins $(18,19)$, an integral process potentially involved in the inflammatory processes associated with Lyme arthritis. In addition, $B$. burgdorferi and T. pallidum spirochetes, as well as lipoproteins and lipopeptides derived from these organisms can induce pro- and anti-inflammatory cytokines, including TNF, IL-1 $\beta$, IL-6, IL-8, IL-10, and IL-12 (16, 18, 19) in monocytes via an initial signaling or binding event with the CD-14 receptor $(16,17)$. Brown et al. (13) observed that mice deficient in IL-10, an anti-inflam- matory cytokine, developed a more severe arthritis when infected with $B$. burgdorferi than did normal mice with the same genetic background. Surprisingly, the numbers of spirochetes in the ankles of the IL-10-deficient mice were tenfold less than in normal mice and their IgG titers to spirochetal antigens were significantly higher. Clearly, the interactions between $B$. burgdorferi and the host's cellular and humoral immune responses are complex, and their effect on the induction and severity of Lyme arthritis requires further investigation.

The abundance of lipoproteins in B. burgdorferi compared to other bacterial pathogens suggests that lipid modification performs an essential role associated with spirochetal framework, activity, or protein function. For example, access of antibody to the exposed loop of the integral outer membrane protein $\mathrm{P} 66$ of $B$. burgdorferi is blocked by the presence of OspA (20). Neither $B$. burgdorferi nor T. pallidum produce true LPS, and analyses of their respective genomes indicate that pathways 
containing LPS-like biosynthetic genes do not exist (6, 7). Both spirochetes lack the ability to elongate longchain fatty acids, so their fatty acid composition reflects that present in the culture medium or host. Lipid moieties of membrane-associated proteins induce immunological stimulation in the mammalian host, similar to LPS in other bacterial pathogens. The lipoprotein-mediated immunostimulatory process can lead to containment and control of inflammatory processes, or in the case of susceptible hosts, Lyme arthritis, with chronicity resulting from low levels of persistent spirochetes.

Variation in outer surface proteins

Both B. burgdorferi and T. pallidum contain genetic systems with the potential for antigenic variation of surface molecules. Borrelia hermsii contains a variable major protein $(\mathrm{Vmp})$ system, which encodes surface-exposed lipoproteins of two size classes, variable small proteins (Vsp) or variable large proteins (Vlp). The Vmp-like system (Vls) recently discovered in B. burgdorferi encodes proteins that are most similar to the Vlp family of $B$. hermsii. B. burgdorferi has 15 silent $v l s$ genes adjacent to an expression site $(v l s E)$, which is found near the telomere of a $28 \mathrm{~kb}$ plasmid (21) (Figure 2). Recombination of $v l s$ genes occurs early during mammalian infection and involves a gene conversion event in which a silent $v l s$ cassette replaces a corresponding region in the $v l s$ gene residing in the $v l s E$ expression site. The $v l s$ system has the potential to produce millions of antigenic variants during infection of the mammalian host (21), and it has been speculated that Vls variation contributes to persistence of the organism in vivo.

Chronic syphilitic infection, seen in untreated individuals where periods of latency are interrupted by episodes of active disease, indicates that these bacteria can evade the host immune system by one or more mechanisms. To date, no antigen has been definitively localized or identified in the outer membrane of T. pallidum, a factor contributing to the concept of the spirochete being a "stealth pathogen." In the genome of $T$. pallidum, a gene family with 12 members, termed $t p r$, has been discovered. These proteins are homologous to the major sheath protein (Msp) of Treponema denticola, a molecule reported to be surface-exposed and involved in cell attachment. Although tpr genes lack a consensus lipoprotein sequence, three members (TprF, TprI, and TprK) are predicted to be associated with the outer membrane of T. pallidum (22). The presence of multiple tprK alleles within the same isolate of T. pallidum and heterogeneity of the tprK gene among isolates (23) suggests that multiple subpopulations or genetic variants exist. The presence of a complex tpr gene family with multiple tprK gene alleles also suggests that tprK has the capacity to undergo recombination with the generation of new alleles or gene variants within an isolate. Because the $\operatorname{tpr} K$ genes are not restricted to a single locus with one promoter, the mechanism for generating gene variation must be different from that used in borreliae at the $v m p$ and $v l s$ loci. However this diversity is generated, tprK gene(s) or other members of the tpr gene family apparently helps explain the lack of cross-immunity in animals challenged with nonhomologous strains (23).

\section{Phenotypic response to changes in temperature}

Temperature is an important environmental cue that triggers adaptation of pathogenic bacteria to their hosts. The ranges in temperature that B. burgdorferi and T. pallidum experience differ significantly. For example, the Lyme disease spirochete experiences considerable change in temperature as it alternates between warmblooded mammals and cold-blooded ticks. In contrast, temperature for T. pallidum is confined to the narrow range imposed on it by human homeostasis. Temperature-induced differential expression of potential virulence determinants has been examined more in $B$. burgdorferi, because this organism can be grown continuously in a liquid, acellular medium and can be analyzed in ticks kept at various ambient temperatures.

Several proven or putative outer surface lipoproteins of B. burgdorferi are differentially expressed at temperatures that correlate with infection of ticks or mammals. During the natural transmission cycle, OspA is produced by the spirochete only in ticks and not in mammals $(11,24,25)$, suggesting that growth at higher temperatures downregulates production of this protein. Supporting this concept are observations that the amount of OspA expressed by the bacteria declines when the spirochetes are cocultivated at mammalian host temperatures with tick cells (26), or when they are present in attached, infected ticks feeding on mice (undergoing warming to near $\left.37^{\circ} \mathrm{C}\right)(24,25)$. The absence of a humoral immune response to OspA in mice and humans infected by tick bite suggests that these OspAnegative spirochetes initiate and persist during infection. In contrast to OspA, OspC is not produced by spirochetes in unfed ticks living at ambient temperature. However, during the first 48 hours that infected ticks attach and feed, spirochetes rapidly upregulate OspC so that $75 \%$ of the spirochetes in the tick midgut express this protein (25). Upregulation of OspC can be reproduced in Kelly's medium by increasing the incubation temperature from $23^{\circ} \mathrm{C}$ to $35-37^{\circ} \mathrm{C}$ (11). Seroconversion to OspC by mammals infected by tick bite supports the presence of OspC on these spirochetes during transmission. The rapid upregulation of OspC during tick feeding suggests that this protein is involved in transmission and early colonization of the spirochete in mammals. These phenotypic changes for OspA and OspC protein expression associated with the natural transmission and maintenance cycle of B. burgdorferi are summarized in Figure 3. Other lipoproteins such as the 
Erps, Mlps, OspE, OspF, and OppAV are also upregulated when spirochetes are grown at higher temperatures $(12,27-29)$, but the expression profile of these proteins in free-living and feeding ticks remains to be determined.

While T. pallidum can be grown in vitro for a limited number of days through cocultivation with cottontail rabbit epithelial (Sf1Ep) cells, this process must be performed at $33^{\circ} \mathrm{C}(30)$. There is circumstantial evidence that T. pallidum varies expression of potential virulence determinants at different temperatures. For example, an exacerbation of syphilitic skin lesions occurs in experimentally infected rabbits with shaved backs when the animals are kept in rooms cooled to $16-18^{\circ} \mathrm{C}$ (5). In addition, the slightly cooler temperature of the human appendages and the occurrence of classic secondary syphilitic skin lesions on the arms and legs suggest that $T$. pallidum grows optimally at a few degrees less than $37^{\circ} \mathrm{C}$.

With regard to high temperature, both B. burgdorferi and $T$. pallidum lack $\sigma$ factor $32(6,7)$, a primary mediator of the heat-shock response, although some evidence exists that $B$. burgdorferi can mount a heat shock response while $T$. pallidum cannot. In vitro cultivation of $B$. burgdorferi at various temperatures demonstrates that the spirochete replicates most quickly at $37^{\circ} \mathrm{C}$. An increase in temperature to $39^{\circ} \mathrm{C}$ retards growth significantly, while a 24 hour exposure at $41^{\circ} \mathrm{C}$ kills all spirochetes in the culture. Therefore, the optimal growth temperature of $B$. burgdorferi is only $4^{\circ} \mathrm{C}$ below the upper lethal limit. The low tolerance of spirochetes for high temperatures is well known and may explain in part the restricted distribution of B. burgdorferi to temperate latitudes and its absence in the tropics, where infected ticks may be exposed to high temperatures detrimental to spirochete survival. Interestingly, the thermal sensitivity of T. pallidum was exploited in the early 1900 s prior to the discovery of penicillin by using fever therapy with malaria or relapsing fever infection to treat patients with general paresis (31).

\section{Closing remarks}

Beyond the morphological and phylogenetic similarities that exist between T. pallidum and B. burgdorferi, the diseases they cause include multistage protean clinical manifestations that include dermatological and neurological involvement and a chronically infected state in the untreated host. Investigators of T. pallidum recognized early the parallels between the syphilis spirochete and the newly emerging tick-transmitted spirochete and contributed their expertise towards understanding borreliae biology and pathogenesis. In fact, the ability to cultivate borreliae in vitro attracted many investigators working on T. pallidum following the discovery of $B$. burgdorferi. The determination of the complete genomes of both B. burgdorferi and T. pallidum $(6,7)$ has already contributed to a greater understanding of chromoso- mal structure, gene regulation, and specific physiological needs, such as the observation that iron is not required for survival $(32,33)$. Comparison of the T. pallidum genome with other bacterial genomes may contribute toward the development of an assemblage of essential nutrients for a medium that will provide continuous growth in vitro. In the absence of such an advance, we can anticipate a continued lag in scientific progress with T. pallidum. Because techniques for inactivating genes have been successful in B. burgdorferi (34) but not yet in T. pallidum, the information gap is likely to grow, but these approaches may provide a framework for future genetic efforts on the syphilis spirochete.

The 0.61 megabase pairs of DNA unique to the $B$. burgdorferi plasmids encode numerous lipoprotein genes, several of which are differentially regulated with temperature. The mechanisms for thermal sensing by spirochetes are not yet known but likely share strategies used by other bacteria (35). Based on expression data, many of the differentially regulated lipoproteins may be involved in adaptation to cycling between coldblooded ticks and warm-blooded vertebrates. Such an extensive repertoire of proteins may be unnecessary for T. pallidum, which does not require an arthropod vector.

One striking difference between these organisms is the ability of T. pallidum to cross the human placenta, resulting in the potentially devastating consequences of congenital syphilis. Transplacental transmission of B. burgdorferi has not been demonstrated in animal studies, and is rare or nonexistent in humans, but transplacental transmission is well documented in the case of $B$. hermsii and other spirochetes that cause relapsing fever. Determination of the genomic sequences of these other pathogens may provide insights into mechanisms responsible for transplacental transmission.

\section{Acknowledgments}

We apologize to colleagues for not citing many pertinent works due to space limitations. We thank Dave Dorward for scanning electron microscopy and Gary Hettrick for graphic art work.

\footnotetext{
1. Quetel, C. 1990. History of syphilis. The Johns Hopkins University Press. Baltimore, Maryland, USA. 342 pp.

2. Burgdorfer, W., et al. 1982. Lyme disease: a tick-borne spirochetosis? Science. 216:1317-1319.

3. Steere, A.C., et al. 1998. Vaccination against Lyme disease with recombinant Borrelia burgdorferi outer-surface lipoprotein A with adjuvant. $N$. Engl. J. Med. 339:209-215.

4. Kelly, R. 1971. Cultivation of Borrelia hermsi. Science. 173:443-444.

5. Turner, T.B., and Hollander, D.H. 1957. Biology of the treponematoses. Monogr. Ser. World Health Organ. 35:9-278.

6. Fraser, C.M., et al. 1997. Genomic sequence of a Lyme disease spirochaete, Borrelia burgdorferi. Nature. 390:580-586.

7. Fraser, C.M., et al. 1998. Complete genome sequence of Treponema pallidum, the syphilis spirochete. Science. 281:375-378, 387-388.

8. Casjens, S., et al. 2000. A bacterial genome in flux: the twelve linear and nine circular extrachromosomal DNAs in an infectious isolate of the Lyme disease spirochete Borrelia burgdorferi. Mol. Microbiol. 35:490-516.
} 
9. Brandt, M.E., Riley, B.S., Radolf, J.D., and Norgard, M.V. 1990. Immunogenic integral membrane proteins of Borrelia burgdorferi are lipoproteins. Infect. Immun. 58:983-991.

10. Chamberlain, N.R., Brandt, M.E., Erwin, A.L., Radolf, J.D., and Norgard, M.V. 1989. Major integral membrane protein immunogens of Treponema pallidum are proteolipids. Infect. Immun. 57:2872-2877.

11. Schwan, T.G., Piesman, J., Golde, W.T., Dolan, M.C., and Rosa, P.A. 1995. Induction of an outer surface protein on Borrelia burgdorferi during tick feeding. Proc. Natl. Acad. Sci. USA. 92:2909-2913.

12. Stevenson, B., Schwan, T.G., and Rosa, P.A. 1995. Temperature-related differential expression of antigens in the Lyme disease spirochete, Borrelia burgdorferi. Infect. Immun. 63:4535-4539.

13. Brown, J.P., Zachary, J.F., Teuscher, C., Weis, J.J., and Wooten, R.M. 1999 Dual role of interleukin-10 in murine Lyme disease: regulation of arthritis severity and host defense. Infect. Immun. 67:5142-5150.

14. Norgard, M.V., Riley, B.S., Richardson, J.A., and Radolf, J.D. 1995. Dermal inflammation elicited by synthetic analogs of Treponema pallidum and Borrelia burgdorferi lipoproteins. Infect. Immun. 63:1507-1515.

15. Morrison, T.B., Weis, J.H., and Weis, J.J. 1997. Borrelia burgdorferi outer surface protein A (OspA) activates and primes human neutrophils. $J$. Immunol. 158:4838-4845.

16. Sellati, T.J., et al. 1999. Activation of human monocytic cells by Borrelia burgdorferi and Treponema pallidum is facilitated by CD14 and correlates with surface exposure of spirochetal lipoproteins. J. Immunol. 163:2049-2056.

17. Wooten, R.M., et al. 1998. The role of CD14 in signaling mediated by outer membrane lipoproteins of Borrelia burgdorferi. J. Immunol. 160:5485-5492.

18. Hirschfeld, M., et al. 1999. Cutting edge: inflammatory signaling by Borrelia burgdorferi lipoproteins is mediated by Toll-like receptor 2 . $J$. Immunol. 163:2382-2386.

19. Lien, E., et al. 1999. Toll-like receptor 2 functions as a pattern recognition receptor for diverse bacterial products. J. Biol. Chem. 274:33419-33425.

20. Bunikis, J., and Barbour, A.G. 1999. Access of antibody or trypsin to an integral outer membrane protein (P66) of Borrelia burgdorferi is hindered by Osp lipoproteins. Infect. Immun. 67:2874-2883.

21.Zhang, J.-R., Hardham, J.M., Barbour, A.G., and Norris, S.J. 1997. Antigenic variation in Lyme disease borreliae by promiscuous recombination of VMP-like sequence cassettes. Cell. 89:275-285.

22. Centurion-Lara, A., et al. 1999. Treponema pallidum major sheath protein homologue TprK is a target of opsonic antibody and the protective immune response. J. Exp. Med. 189:647-656.

23. Centurion-Lara, A., Godornes, C., Castro, C., Van Voorhis, W.C., and Lukehart, S.A. 2000. The tprK gene is heterologous among Treponema pallidum strains and has multiple alleles. Infect. Immun. 68:824-831.

24. de Silva, A.M., Telford, S.R., III, Brunet, L.R., Barthold, S.W., and Fikrig, E. 1996. Borrelia burgdorferi OspA is an arthropod-specific transmissionblocking Lyme disease vaccine. J. Exp. Med. 183:271-275.

25. Schwan, T.G., and Piesman, J. 2000. Temporal changes in outer surface proteins A and $\mathrm{C}$ of the Lyme disease-associated spirochete, Borrelia burgdorferi, during the chain of infection in ticks and mice. J. Clin. Microbiol. 38:383-388.

26. Obonyo, M., Munderloh, U.G., Fingerle, V., Wilske, B., and Kurtti, T.J. 1999. Borrelia burgdorferi in tick cell culture modulates expression of outer surface proteins $\mathrm{A}$ and $\mathrm{C}$ in response to temperature. J. Clin. Microbiol. 37:2137-2141.

27. Porcella. S.F., Fitzpatrick, C.A., and Bono, J.L. 2000. Expression and immunological analysis of the plasmid-encoded $m l p$ genes of Borrelia burgdorferi strain B31. Infect. Immun. 68:4992-5001.

28. Bono, J.L., Tilly, K., Stevenson, B., Hogan, D., and Rosa, P. 1998. Oligopeptide permease in Borrelia burgdorferi: putative peptide-binding components encoded by both chromosomal and plasmid loci. Microbiology. 144:1033-1044.

29. Stevenson, B., Bono, J.L., Schwan, T.G., and Rosa, P. 1998. Borrelia burgdorferi Erp proteins are immunogenic in mammals infected by tick bite, and their synthesis is inducible in cultured bacteria. Infect. Immun. 66:2648-2654.

30. Fieldsteel, A.H., Cox, D.L., and Moeckli, R.A. 1981. Cultivation of virulent Treponema pallidum in tissue culture. Infect. Immun. 32:908-915.

31. Solomon, H.C., and Kopp, I. 1937. Fever therapy. N. Engl. J. Med. 217:805-814.

32. Posey, J.E., Hardham, J.M., Norris, S.J., and Gherardini, F.C. 1999. Characterization of a monganese-dependent regulatory protein, TroR, from Treponema pallidum. Proc. Natl. Acad Sci. USA. 96:10887-10892.

33. Posey, J.E., and Gherardini, F.C. 2000. Lack of a role for iron in the Lyme disease pathogen. Science. 288:1651-1653.

34. Bono, J.L., et al. 2000. Efficient targeted mutagenesis in Borrelia burgdorferi. J. Bacteriol. 182:2445-2452.

35. Hurme, R., and Rhen, M. 1998. Temperature sensing in bacterial gene regulation: what it all boils down to. Mol. Microbiol. 30:1-6. 